\title{
Are large high-altitude electric fields caused by global thunderstorms?
}

\author{
Valery V. Plotkin \\ Institute of Geophysics, Siberian Branch RAS, 630090 Novosibirsk, Russia \\ (Received June 14, 2000; Revised December 19, 2001; Accepted December 19, 2001)
}

\begin{abstract}
Using the model of a spherical symmetric atmosphere the large-scale electric fields generated by the global thunderstorms are investigated. Theoretical formulations have been developed to calculate high-altitude electric fields due to global thunderstorms, taking into account the presence of the ionosphere. We make use of the assumption that in the ionosphere the geomagnetic field lines are equipotentials. The calculations indicate that the solution is highly sensitive to the variation of this upper boundary condition.
\end{abstract}

\section{Introduction}

Kuznetsov et al. (1990) have shown, that there is a universal variation of the F2-layer critical frequency of the ionosphere which is similar to the known universal variation of the global atmospheric electric field near the Earth's surface (Volland, 1984). To understand the origin of the $\mathrm{f}_{0} \mathrm{~F} 2$ universal variation it is important to know the value of a high-altitude electric field produced in the ionosphere by global thunderstorms, located in the lower atmosphere, and how significantly it affects ionospheric processes. The available models of the global atmospheric electric circuit either assume an equipotential ionosphere (Makino and Ogawa, 1984), or north-south symmetry of the electric potential in the ionosphere due to equipotential geomagnetic field lines (Hays and Roble, 1979). The correct upper boundary condition for an atmospheric electric field (Kuznetsov et al., 1995 ) is necessary. To specify the upper boundary condition we use the fact that in the ionosphere the longitudinal conductivity greatly exceeds other components of the conductivity tensor. Therefore the magnitude of the projection of the electric field along the direction of the geomagnetic field lines tends to zero.

It is known that due to good conductivity of these lines the electric field of separate powerful thunderstorms (Park and Dejnakarintra, 1973; Velinov and Tonev, 1993) can be transferred into the ionosphere and magnetosphere. To simulate the ionospheric response due to such thunderstorm source at altitude of $150 \mathrm{~km}$, the vertical component of a field was equated to zero by Park and Dejnakarintra (1973). We have used a similar approximation, in accordance with model of Makino and Ogawa (1984), to obtain preliminary estimates of the electric field in the ionosphere caused by global thunderstorms, which were taken.

However for global modelling the specified boundary condition of equipotential geomagnetic field lines only approximately reflects the situation at high- and mid-latitudes

Copy right(c) The Society of Geomagnetism and Earth, Planetary and Space Sciences (SGEPSS); The Seismological Society of Japan; The Volcanological Society of Japan; The Geodetic Society of Japan; The Japanese Society for Planetary Sciences. and obviously is not correct at low-latitudes near equator. So, this paper also submits results for the case when the longitudinal component of the electric field is equated to zero.

The case of a simple exponential high-altitude dependence of atmospheric conductivity was investigated first. Then it is of interest to study how changing the high-altitude profile of the atmospheric conductivity in the region the Earth-Ionosphere effects the ionospheric potential caused by global thunderstorms.

In the model of Makino and Ogawa (1984) it is supposed that horizontal electric currents and fields are not present in the atmosphere. In another work (Makino and Takeda, 1984) the current density distribution in the ionosphere was studied by considering vertical upward currents from the atmosphere and also the electric fields generated in the ionosphere were calculated. In these calculations the altitude of the ionosphere is taken to be $90 \mathrm{~km}$ and the difference between maximum and minimum of potential in the ionosphere did not exceed $55 \mathrm{~V}$. However when the altitude of the ionosphere was increased to $105 \mathrm{~km}$ (Hays and Roble, 1979) the value of potential difference becomes $1600 \mathrm{~V}$.

Such a change is explained by Makino and Takeda (1984) as due to differences in the models of ionospheric conductivity. We are going to show that the integrated atmospheric conductivity has a more important influence on the value of the potential difference generated in the ionosphere by global thunderstorms.

\section{Calculation Method}

Away from the geomagnetic equator for a rough estimates one can consider, (Volland, 1984; Park and Dejnakarintra, 1973), that in the ionosphere the vertical (radial) component of the electric field $\vec{E}$ tends to zero rather than the longitudinal component. For simplicity, considering the global distribution of thunderstorm sources in a spherical symmetric atmosphere, it is possible to write the electric potential as (Volland, 1984):

$$
\operatorname{div}(\sigma(r) \nabla u)=J_{p}(\theta, \lambda)\left[\delta\left(r-r_{+}\right)-\delta\left(r-r_{-}\right)\right],
$$


where $u$ is the potential, $\sigma$ is the conductivity, $J_{p}$ is the intensity of current generators, $r, \theta$ and $\lambda$ are spherical coordinates with origin at the center of the Earth and $\delta(r)$ is the Dirac function. We consider $\vec{E}$ given by $\vec{E}=-\nabla u$. We assume also (Hays and Roble, 1979), that thunderstorm extraneous currents are located in a thin spherical layer as a distribution of vertical dipole current generators with positive sources of the current located above the layer in points $r_{+}(\theta, \lambda)$ and with negative ones located below in points $r_{-}(\theta, \lambda)$, and both kind of sources have identical intensity $J_{p}(\theta, \lambda)$. We use the expression for conductivity in isotropic medium $\left(\sigma=\sigma_{0}=\sigma_{P}\right.$, where $\sigma_{0}$ and $\sigma_{P}$ are specific and Pedersen conductivities, respectively).

As a further simplification, it is possible to accept, that the altitudes of positive and negative sources of the current do not depend on co-latitude $\theta$ and longitude $\lambda$. Then from Eq. (1), expanding $u(\theta, \lambda)$ and $J_{p}(\theta, \lambda)$ into the series of spherical harmonics $Y_{m n}(\theta, \lambda)$,

$$
\begin{aligned}
& J_{p}(\theta, \lambda)=\sum_{n=0}^{\infty} \sum_{m=-n}^{m=n} \bar{J}_{p m n} Y_{m n}(\theta, \lambda), \\
& u(r, \theta, \lambda)=\sum_{n=0}^{\infty} \sum_{m=-n}^{m=n} \bar{u}_{m n} Y_{m n}(\theta, \lambda),
\end{aligned}
$$

we obtain equations defining $\bar{u}_{m n}(r)$ (in the approximation of a thin layer $\Delta r \ll R_{E}$, where $R_{E}$ is radius of the Earth and $\left.\triangle r=r-R_{E}\right)$ :

$$
\begin{gathered}
\frac{d^{2} \bar{u}_{m n}}{d r^{2}}+\frac{1}{\sigma} \frac{d \sigma}{d r} \frac{d \bar{u}_{m n}}{d r}-\frac{n(n+1)}{R_{E}^{2}} \bar{u}_{m n} \\
=\bar{J}_{p m n}\left[\frac{\delta\left(r-r_{+}\right)}{\sigma\left(r_{+}\right)}-\frac{\delta\left(r-r_{-}\right)}{\sigma\left(r_{-}\right)}\right] .
\end{gathered}
$$

From here we are employing the convention which we shall continue to use throughout: all radial distances $r$ are the altitudes above the Earth's surface. The lower boundary condition at $r=0$ (infinite surface conductivity is assumed) is $\bar{u}_{m n}=0$. The upper boundary condition is $\frac{d \bar{u}_{m n}}{d r}=0$ at altitude $r=H$, where the geomagnetic field lines may be treated as equipotentials.

It is not difficult to find the solution of (3) under the indicated boundary conditions in the case, when $\sigma(r)=$ $\sigma_{0} \exp (2 \gamma r), \quad \gamma=$ const. In particular, for the potential in the region located higher than the sources, it turns out that

$$
\begin{aligned}
\bar{u}_{m n}(r)= & \left\{\frac{f_{+}}{\alpha_{1}-\alpha_{2}}\left(e^{-\alpha_{2} r_{+}}-e^{-\alpha_{1} r_{+}}\right)\right. \\
& \left.+\frac{f_{-}}{\alpha_{1}-\alpha_{2}}\left(e^{-\alpha_{2} r_{-}}-e^{-\alpha_{1} r_{-}}\right)\right\} \times S(r), \\
S(r)= & \frac{\alpha_{1} e^{\alpha_{1} H+\alpha_{2} r}-\alpha_{2} e^{\alpha_{2} H+\alpha_{1} r}}{\alpha_{1} e^{\alpha_{1} H}-\alpha_{2} e^{\alpha_{2} H}} .
\end{aligned}
$$

Here

$$
\begin{gathered}
f_{+}=\frac{\bar{J}_{p m n}}{\sigma\left(r_{+}\right)}, \quad f_{-}=-\frac{\bar{J}_{p m n}}{\sigma\left(r_{-}\right)}, \\
p^{2}=\frac{n(n+1)}{R_{E}^{2}}, \\
\alpha_{1}=-\gamma-\sqrt{\gamma^{2}+p^{2}}, \\
\alpha_{2}=-\gamma+\sqrt{\gamma^{2}+p^{2}} .
\end{gathered}
$$

It is possible to generalize this solution for a medium described by arbitrary piecewise exponential high-altitude (radial) functions $\sigma(r)$. Let us assume, that in the $i$ th layer,

$$
\begin{aligned}
& \sigma(r)=\sigma_{i-1} e^{2 \gamma^{(i)}\left(r-r_{i-1}\right)} \\
& r_{i-1} \leq r \leq r_{i}, \quad i=1,2, \ldots, I
\end{aligned}
$$

Here $r_{0}=0$ (the Earth's surface), $\sigma_{i}=\sigma\left(r_{i}\right), \gamma^{(i)}=$ $\frac{1}{2\left(r_{i}-r_{i-1}\right)} \ln \frac{\sigma_{i}}{\sigma_{i-1}}, r_{I}$ is the altitude of the upper boundary condition. The solution of (3) in the $i$ th layer $r_{i-1} \leq r \leq r_{i}$ is

$$
\begin{aligned}
& \bar{u}_{m n}(r)= C_{1 m n}^{(i)} \exp \left[\alpha_{1 m n}^{(i)}\left(r-r_{i-1}\right)\right] \\
&+C_{2 m n}^{(i)} \exp \left[\alpha_{2 m n}^{(i)}\left(r-r_{i-1}\right)\right], \\
& \alpha_{1 m n}^{(i)}=-\gamma^{(i)}-\sqrt{\gamma^{(i)^{2}}+p_{m n}^{2}}, \\
& \alpha_{2 m n}^{(i)}=-\gamma^{(i)}+\sqrt{\gamma^{(i)^{2}}+p_{m n}^{2}}, \\
& p_{m n}^{2}=\frac{n(n+1)}{R_{E}^{2}} .
\end{aligned}
$$

It is required to match the solutions (6) at the boundaries of layers (5) and at altitudes of the sources $r_{+}$and $r_{-}$. This matching gives the relations between constants $C_{1,2 m n}^{(1)}$ in the lower layer and $C_{1,2 m n}^{(I)}$ in the upper layer as

$$
\begin{aligned}
\vec{C}_{m n}^{(I)}= & \hat{M}^{(I-1)} \ldots \hat{M}^{(1)} \vec{C}_{m n}^{(1)} \\
& +\hat{M}^{(I-1)} \ldots \hat{M}^{(2)} \vec{\Phi}_{1}+\ldots \\
& \left.+\hat{M}^{(I-1)} \vec{\Phi}_{I-2}+\vec{\Phi}_{I-1}\right) .
\end{aligned}
$$

Here $\vec{C}_{m n}^{(i)}=\left\{C_{1 m n}^{(i)}, C_{2 m n}^{(i)}\right\}, \vec{\Phi}_{i}=\{1,-1\} \Phi_{i}, \Phi_{i}$ is non-zero only at the levels of sources. Also $\Phi_{i_{+}}=\frac{\bar{J}_{p m n}}{\sigma\left(r_{+}\right)} \cdot \frac{1}{\alpha_{1 m n}^{(i++1)}-\alpha_{2 m n}^{(i+1)}}$ at $r_{i}=r_{i_{+}}=r_{+}$and $\Phi_{i_{-}}=-\frac{\bar{J}_{p m n}}{\sigma\left(r_{-}\right)} \cdot \frac{1}{\alpha_{1 m n}^{\left(i_{-}+1\right)}-\alpha_{2 m n}^{\left(i_{-}+1\right)}}$ at $r_{i}=r_{i_{-}}=r_{-}$. Matrix $\hat{M}^{(i)}$ has the form:

$$
\hat{M}^{(i)}=\left(\begin{array}{ll}
\hat{M}_{11}^{(i)} & \hat{M}_{12}^{(i)} \\
\hat{M}_{21}^{(i)} & \hat{M}_{22}^{(i)}
\end{array}\right)
$$

with

$$
\begin{aligned}
& \hat{M}_{11}^{(i)}=\frac{\alpha_{2 m n}^{(i+1)}-\alpha_{1 m n}^{(i)}}{\alpha_{2 m n}^{(i+1)}-\alpha_{1 m n}^{(i+1)}} e^{\alpha_{1 m n}^{(i)}\left(r_{i}-r_{i-1}\right)}, \\
& \hat{M}_{12}^{(i)}=\frac{\alpha_{2 m n}^{(i+1)}-\alpha_{2 m n}^{(i)}}{\alpha_{2 m n}^{(i+1)}-\alpha_{1 m n}^{(i+1)}} e^{\alpha_{2 m n}^{(i)}\left(r_{i}-r_{i-1}\right)}, \\
& \hat{M}_{21}^{(i)}=\frac{\alpha_{1 m n}^{(i+1)}-\alpha_{1 m n}^{(i)}}{\alpha_{1 m n}^{(i+1)}-\alpha_{2 m n}^{(i+1)}} e^{\alpha_{1 m n}^{(i)}\left(r_{i}-r_{i-1}\right)}, \\
& \hat{M}_{22}^{(i)}=\frac{\alpha_{1 m n}^{(i+1)}-\alpha_{2 m n}^{(i)}}{\alpha_{1 m n}^{(i+1)}-\alpha_{2 m n}^{(i+1)}} e^{\alpha_{2 m n}^{(i)}\left(r_{i}-r_{i-1}\right)} .
\end{aligned}
$$

As the upper boundary condition the longitudinal component of electric field was set to zero.

$$
\begin{aligned}
& \cos \chi \frac{\partial u}{\partial r}+\sin \chi\left(\cos D \frac{\partial u}{R_{E} \partial \theta}\right. \\
& \left.+\sin D \frac{\partial u}{R_{E} \sin \theta \partial \lambda}\right)=0, \quad r=r_{I} .
\end{aligned}
$$


Here $\chi$ is the angle between the magnetic field of the Earth and the radial distance, $D$ is magnetic declination at the point of observation. We used for the geomagnetic field a model of a central inclined magnetic dipole with coordinates of the north geomagnetic pole $\theta_{0}=11.5^{\circ}$ and $\lambda_{0}=291^{\circ}$. Let us substitute in (8) the solution (6) for the upper layer to obtain

$$
\begin{aligned}
& \sum_{m n} C_{1 m n}^{(I)}\left[\cos \chi \alpha_{1 m n}^{(I)} e^{\alpha_{1 m n}^{(I)}\left(r_{I}-r_{I-1}\right)} Y_{m n}(\theta, \lambda)\right. \\
& +\sin \chi e^{\alpha_{1 m n}^{(I)}\left(r_{I}-r_{I-1}\right)}\left(\cos D \frac{\partial Y_{m n}(\theta, \lambda)}{R_{E} \partial \theta}\right. \\
& \left.\left.+\sin D \frac{\partial Y_{m n}(\theta, \lambda)}{R_{E} \sin \theta \partial \lambda}\right)\right] \\
& +\sum_{m n} C_{2 m n}^{(I)}\left[\cos \chi \alpha_{2 m n}^{(I)} e^{\alpha_{2 m n}^{(I)}\left(r_{I}-r_{I-1}\right)} Y_{m n}(\theta, \lambda)\right. \\
& +\sin \chi e^{\alpha_{2 m n}^{(I)}\left(r_{I}-r_{I-1}\right)}\left(\cos D \frac{\partial Y_{m n}(\theta, \lambda)}{R_{E} \partial \theta}\right. \\
& \left.\left.+\sin D \frac{\partial Y_{m n}(\theta, \lambda)}{R_{E} \sin \theta \partial \lambda}\right)\right]=0 .
\end{aligned}
$$

Taking into account expressions (7) and the lower boundary condition $u=0$ at $r=0$, from which follows that $C_{1 m n}^{(1)}=-C_{2 m n}^{(1)}=C_{m n}$, we obtain, instead of (9), an equation involving amplitudes of spherical harmonics $C_{m n}$, which determine the required solution. Using numerical approximation, such an equation was written for each of the mesh points on the sphere. The redefined system of equations dependent on amplitudes $C_{m n}$ was solved by the method of least squares. We used a $2^{\circ}$ grid in latitude and longitude (16380 equations) and the expansion in spherical functions for orders $n \leq 8$ (81 unknown amplitudes $C_{m n}$ ).

\section{Results and Discussion}

At first we consider the properties of the solution given by (4) with the upper boundary condition as $\frac{\partial u}{\partial r}=0$. For a spherical symmetric mode ( $m=n=0)$ this solution coincides with another obtained by Volland (1984). Putting $r=$ $H$ gives the global potential of the ionosphere as a whole. However the solution given by (4) for large-scale harmonics with small $n$, when $\alpha_{2} \exp \left(\alpha_{2} H\right) \ll \alpha_{1} \exp \left(\alpha_{1} H\right)$ is similar to results obtained by Park and Dejnakarintra (1973) (see figure 7 in this work). In Fig. 1 curves describing the highaltitude coefficients $S(r)$ in (4) at $n=1$ depending on various values of parameter $H$ (there are shown the numbers at curves) are given. As is evident from Fig. 1, at $r=H$ the amplitudes of large-scale harmonics with small $n$ strongly depend on the value $H$. It means that the solution given by (4) can be highly sensitive to variation of this value.

Figure 2(a) shows the total contribution of all spherical harmonics to the ionospheric potential at $r=H$. The simulation used the following values of parameters $(2 \gamma=$ $\left.1 / 6 \mathrm{~km}^{-1}, R_{E}=6371 \mathrm{~km}, r_{+}=7.5 \mathrm{~km}, r_{-}=5.5 \mathrm{~km}\right)$, which are close to ones used by Volland (1984) and Makino and Ogawa (1984). The global distribution of thunderstorm sources (Fig. 2(b), contours a $200 \mathrm{pA} / \mathrm{m}^{2}$ intervals) is for 19 UT according to the model of Makino and Ogawa (1984). In thunderstorm areas initial currents and electric fields are

\section{$\mathrm{r}, \mathrm{km}$}

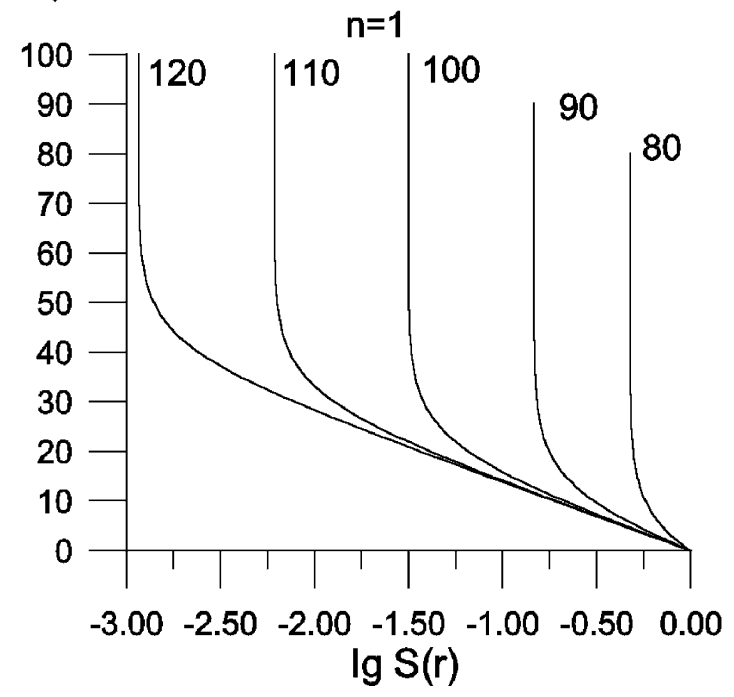

Fig. 1. The high-altitude factor $S(r)$ of amplitude attenuation of potential large-scale spherical harmonic $(n=1)$ excited in atmosphere by thunderstorm global sources at 19 UT. The numbers next to the curves are the altitudes in kilometers where the upper boundary condition is equivalent to the assumption of equipotential geomagnetic field lines.

directed vertically upwards. The electric field on the surface of the Earth (Fig. 2(c)) corresponds to the model of Makino and Ogawa (1984). The difference between a maximum and minimum of potential in the ionosphere gives a value of 3.7 $\mathrm{kV}$ for $H=120 \mathrm{~km}$, whereas for $H=110 \mathrm{~km}$ it reaches $19 \mathrm{kV}$ and for $H=100 \mathrm{~km}$ it is already $102 \mathrm{kV}$. It follows from this, that for $H=100 \mathrm{~km}$ horizontal components of electric field can reach values of $1 \mathrm{mV} / \mathrm{m}$. An electric field of such a value basically can cause appreciable disturbances in $\mathrm{f}_{0} \mathrm{~F} 2$ (Hegai and Kim, 1990). In this connection the universal variation of the atmospheric electric field near the Earth's surface could cause a similar universal variation of $\mathrm{f}_{0} \mathrm{~F} 2$. However the large differences caused by different reasonable values of $H$ make it difficult to be conclusive about this effect.

The upper boundary condition of $\frac{\partial u}{\partial r}=0$ at $r=r_{I}$ reflects the upper boundary condition in the form of (8) only at small $\chi$. Therefore it is of interest to compare results of simulation using the upper boundary condition of $\frac{\partial u}{\partial r}=0$ and the one in the form of (8). Let us note at once, that the form of the upper boundary condition has a small effect on the value and character of distribution of the electric field on the Earth's surface (at $r=0$ ). Changing the upper boundary condition essentially affects the distribution of ionospheric potential caused by global thunderstorms. As proof we show results of simulations of electric potential in the ionosphere with the upper boundary condition in the form of (8) (Fig. 3(a)) and as $\frac{\partial u}{\partial r}=0$ (Fig. 3(b)). The simulations were executed for the following values of parameters: $2 \gamma=1 / 7 \mathrm{~km}^{-1}, R_{E}=6371 \mathrm{~km}, r_{+}=7.5 \mathrm{~km}, r_{-}=5.5$ $\mathrm{km}$ and $H=100 \mathrm{~km}$. Note that in the case when $2 \gamma=1 / 6$ $\mathrm{km}^{-1}$ (Fig. 2(a)), the average value of ionospheric potential is of $487 \mathrm{kV}$, while if $2 \gamma=1 / 7 \mathrm{~km}^{-1}$ it is increased to $550 \mathrm{kV}$ (Fig. 3(b)), since in the latter case the atmosphere columnar resistance between the Earth's surface and 

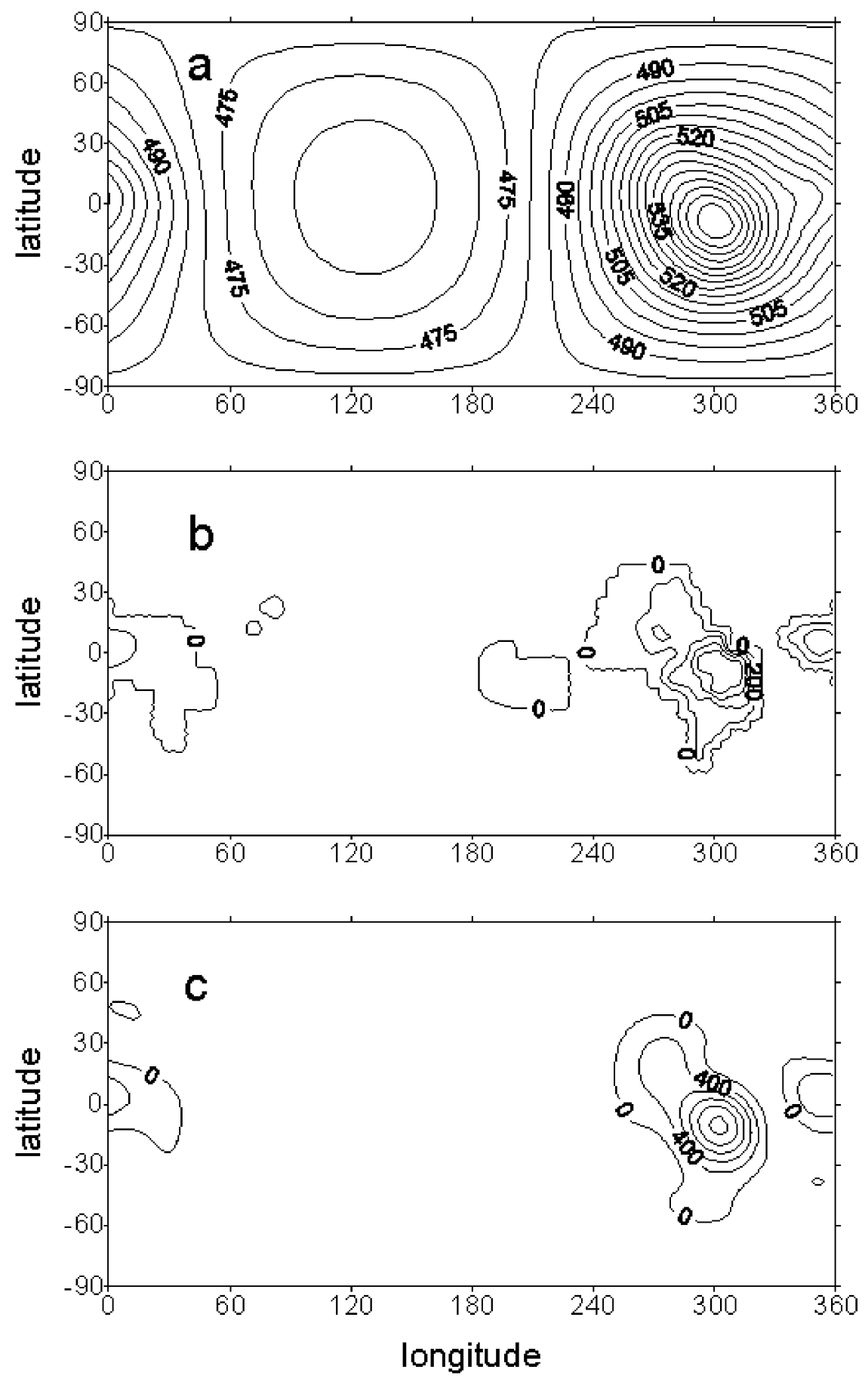

Fig. 2. The electric potential (levels through $5 \mathrm{kV}$ ) (a) excited in the ionosphere by thunderstorm global currents with initial distribution (b) at 19 UT (levels through $200 \mathrm{pA} / \mathrm{m}^{2}$ ). Initial currents and electric fields in thunderstorm areas are directed vertically upwards. The electric field on the surface of the Earth (levels through $400 \mathrm{~V} / \mathrm{m}$ ) (c) corresponds to model (Makino and Ogawa, 1984).

the ionosphere is greater. Then the same amplitude of current generator produces a greater potential. A distribution of initial thunderstorm currents generating the field, similar to the one given in Fig. 2(b) (time is 19 UT) was used. One can see that the averaged potential of the ionosphere depends also on the form of the upper boundary condition and changes from $550 \mathrm{kV}$ (Fig. 3(b)) to $570 \mathrm{kV}$ (Fig. 3(a)). With the upper boundary condition in the form of (8) it is evident that the difference between maximum and minimum values of ionospheric potential becomes noticeably less, other things being equal. With the upper boundary condition in the form of (8) the potential distribution in the ionosphere is more structured than just a simple maximum above the thunderstorm area (Fig. 3(b)). Figure 3(a) shows that above a thunderstorm source in the ionosphere there is a structure in the form of two areas of increased potential separated by a minimum. This is similar to the results of Pulinets et al. (1998), who considered how atmospheric anomalies in electric field near the Earth's surface influence the ionosphere.

The value of the electric field, penetrating from below, 

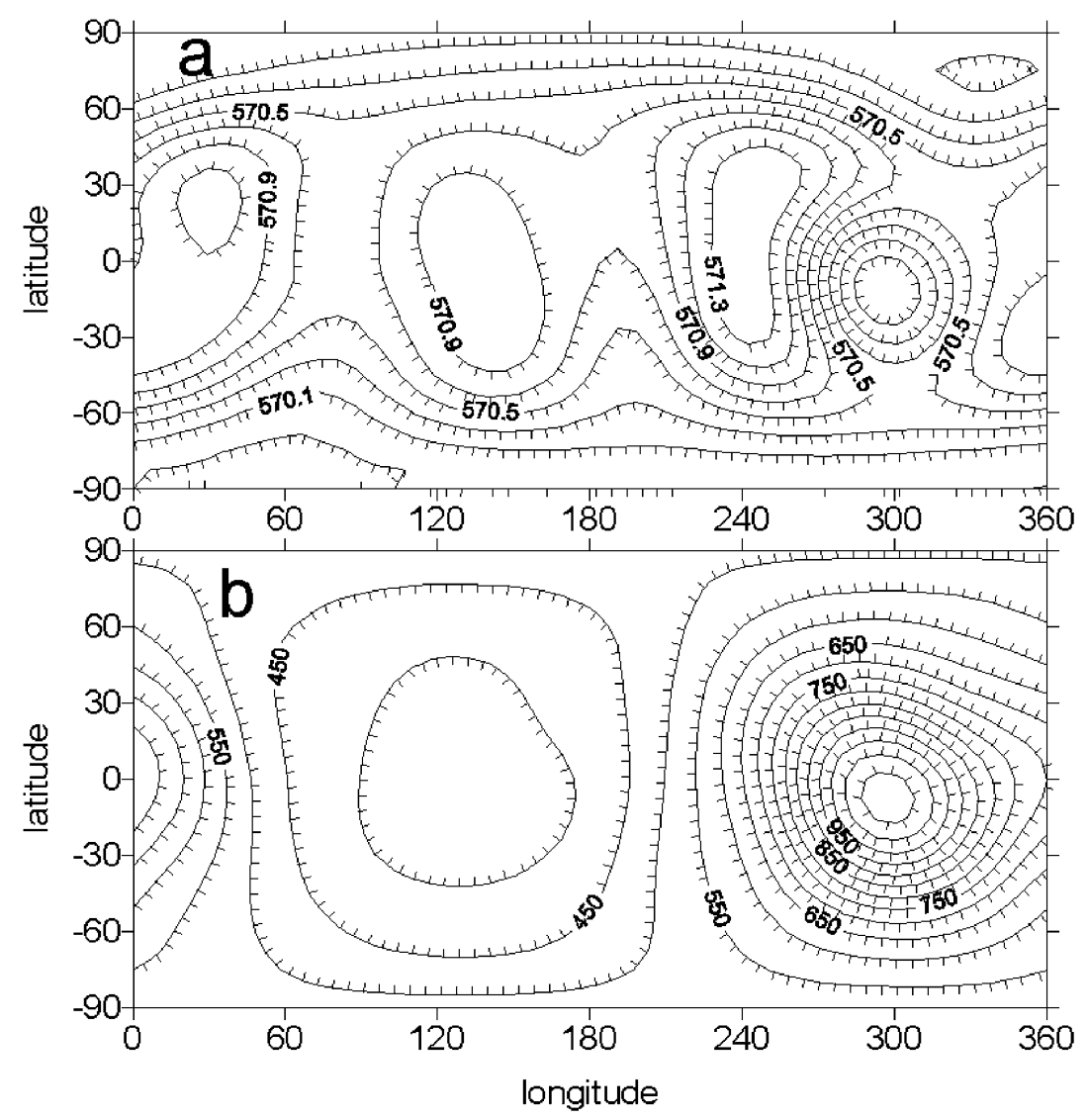

Fig. 3. The electric potential excited in the ionosphere by thunderstorm global currents with initial distribution (Fig. 2(b)) with the upper boundary condition in the form of (8) (levels through $0.2 \mathrm{kV}$ ) (a) and as $\frac{\partial u}{\partial r}=0$ (b) (levels through $50 \mathrm{kV}$ ).
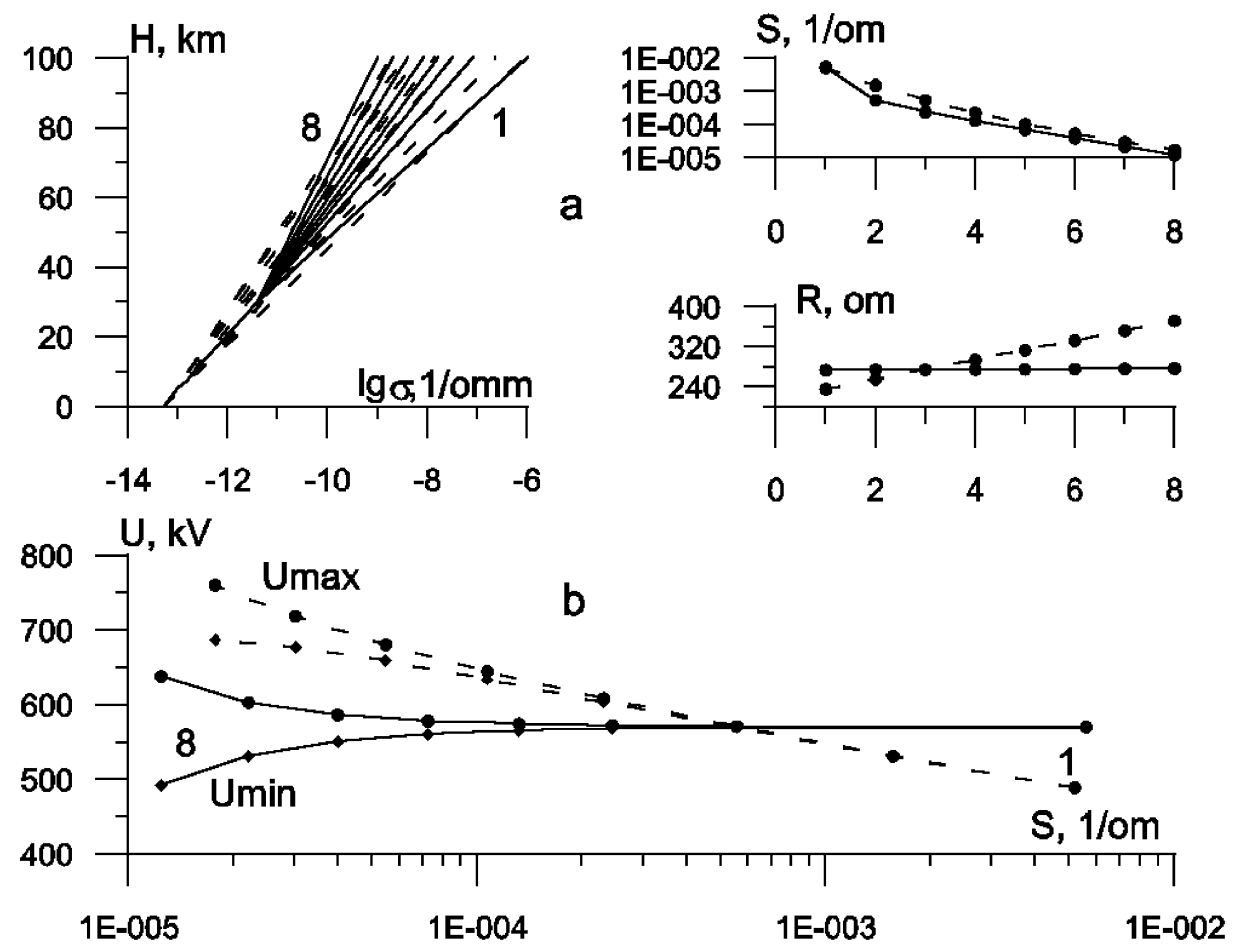

Fig. 4. The conductivity profiles and their integrated conductivity $S$ and resistance $R$ of the atmosphere (a). The profiles are numbered from 1 up to 8 (from right to the left). Maximal $U_{\max }$ and minimal $U_{\min }$ values of electric potential in the ionosphere are given for different integrated conductivity of the atmosphere (b). They are shown by dotted and continuous lines for profiles represented analogous. 
essentially depends on the integrated conductivity $(S=$ $\left.\int_{0}^{H} \sigma(z) d z\right)$ and resistance $\left(R=\frac{1}{4 \pi R_{E}^{2}} \int_{0}^{H} \frac{d z}{\sigma(z)}\right)$ of the atmosphere. In Fig. 4 results of simulations of electric potential in the ionosphere for several conductivity profiles, given in the top half of Fig. 4, are presented. The value of integrated resistance $R$ and conductivity $S$ of the atmosphere for these profiles are also given here. The profiles are numbered from 1 up to 8 (from right to the left). It is apparent that for profiles given by continuous lines, the resistance of the atmosphere remains practically the same, because it is determined most of all by resistance of the lower layers with height $\leq 30 \mathrm{~km}$. The resistance of the atmosphere for profiles represented by dotted lines increases from the first to the eighth profile. Integrated conductivity $S$ is determined by the conductivity of the upper layers of the atmosphere. It is evident that the conductivity $S$ varies approximately in the same manner for both systems of profiles, decreasing from the first to the eighth profile. In the bottom part of Fig. 4 maximal $U_{\max }$ and minimal $U_{\min }$ values of electric potential in the ionosphere are given for different integrated conductivity $S$ of the atmosphere. They are shown by dotted and continuous lines for profiles represented analogous. Thus profile 3 of the first group coincides with profile 2 of the second one. The average value of ionospheric potential depends on the resistance $R$ of the atmosphere. Increasing $R$ leads to growing averaged ionospheric potential. In the upper layers of an atmosphere the vertical currents spread horizontally and their integrated conductivity $S$ begins to play the leading role. With reduction of $S$ the difference between $U_{\max }$ and $U_{\min }$ is increased and can become appreciable, below $S \sim 10^{-4}$ mho (profiles $4-8$, located in the top left part of Fig. 4(a)). The considered profiles of conductivity are close enough to real night-time profiles (Park and Dejnakarintra, 1973). Therefore it is possible to expect, that at night, and also in the case of an abnormal decrease of conductivity (for example, during Forbush-decrease), the high-altitude electric fields, generated by global thunderstorms will reach appreciable values. Note that the universal variation of the atmospheric electric field was measured at $26-\mathrm{km}$ altitude by Holzworth et al. (1984) and was established in mesosphere by Zadorozhny and Tyutin (1997).

\section{Conclusion}

The results of simulations of large-scale electric fields generated by global thunderstorms demonstrate the possible penetration of these fields, with appreciable amplitudes, to ionospheric altitudes. This points to a possible casual relationship between universal variations of the atmospheric electric field and $\mathrm{f}_{0} \mathrm{~F} 2$. However the solution is highly sensi- tive to variations in the altitude at which the geomagnetic field lines may be treated as equipotentials. The electric field penetrating from below essentially depends on the integrated conductivity and resistance of the atmosphere. The large differences caused by differences in reasonable upper boundary conditions make it difficult to be conclusive about the magnitudes of high-altitude global thunderstorm electric fields. Therefore numerical simulations of high-altitude electric field generated by the global thunderstorms in an atmosphere with more realistic properties, correctly taking into account the influence of the anisotropy conductivity, are necessary.

Acknowledgments. This work was supported financially by RFBR (grant N 99-05-64676).

\section{References}

Hays, P. B. and R. G. Roble, A quasi-static model of global atmospheric electricity. 1. The lower atmosphere, J. Geophys. Res., 84, 3291-3305, 1979.

Hegai, V. V. and V. P. Kim, The formation of a cavity in the night-time midlatitude ionospheric E-region above a thundercloud, Planet. Space Sci., 38, 703-707, 1990.

Holzworth, R. H., T. Onsager, P. Kintner, and S. Powell, Planetary-scale variability of the fair-weather vertical electric field in the stratosphere, Phys. Review Lett., 53, 1398-1401, 1984.

Kuznetsov, V. V., V. V. Plotkin, I. I. Nesterova, and M. S. Pozdeeva, Universal diurnal variation of F2-layer critical frequency, J. Geomag. Geoelectr., 42, 1237-1240, 1990.

Kuznetsov, V. V., V. V. Plotkin, N. I. Izraileva, and I. I. Nesterova, The investigation of electromagnetic interactions between regions surrounding the Earth-1. Initial equations, the boundary conditions, the distribution of external currents and the methods of numerical solution, J. Atmos. Terr. Phys., 57, 1639-1646, 1995.

Makino, M. and T. Ogawa, Responses of atmospheric electric field and airearth current to variations of conductivity profiles, J. Atmos. Terr. Phys., 46, 431-445, 1984.

Makino, M. and M. Takeda, Three-dimensional ionospheric currents and fields generated by the atmospheric global circuit current, J. Atmos. Terr. Phys., 46, 199-206, 1984.

Park, C. G. and M. Dejnakarintra, Penetration of thundercloud electric fields into the ionosphere and magnetosphere. 1. Middle and subauroral latitudes, J. Geophys. Res., 78, 6623-6633, 1973.

Pulinets, S. A., V. V. Hegai, K. A. Bojarchuk, and A. M. Lomonosov, Atmospheric electric field as a source of variability of the ionosphere, Usp. Phys. Nauk., 168, 582-589, 1998 (in Russian).

Velinov, P. and P. Tonev, Penetration of horizontal and vertical components of thundercloud electric fields into the ionosphere-modelling and analysis, Bulgarian Geophys. Journ., 19, 64-72, 1993.

Volland, H., Atmospheric Electrodynamics, 205 pp., Springer-Verlag, Berlin, 1984.

Zadorozhny, A. M. and A. A. Tyutin, Universal diurnal variation of mesospheric electric fields, Adv. Space Res., 20, 2177-2180, 1997.

V. V. Plotkin (e-mail: plotkin@uiggm.nsc.ru) 